\title{
LIDERAZGO Y AUTOEVALUACIÓN INSTITUCIONAL
}

LIDERANÇA E AUTO-AVALIAÇÃO INSTITUCIONAL

\section{LEADERSHIP AND INSTITUTIONAL SELF-EVALUATION}

\author{
Alejandro BERRIOS ${ }^{1}$ \\ Margarita ARAVENA ${ }^{2}$ \\ Marina GARCÍA-CARMONA ${ }^{3}$ \\ Mario MARTÍN BRIS ${ }^{4}$
}

RESUMEN: Este trabajo describe la influencia positiva del liderazgo en los procesos de autoevaluación institucional en una organización que busca levantar un diagnóstico de gestión participativa propiciando la colaboración de todos los agentes involucrados, cuyo propósito es subsanar las debilidades y necesidades detectadas para luego tomar decisiones distribuidas, colegiadas y consensuadas en beneficio de las personas y por ende de la organización. Para conseguir la gestión participativa, es necesario que la institución cuente con una metodología para autoevaluarse y por medios de técnicas cualitativas y cuantitativas es posible implementarla periódicamente según la planificación estratégica y de los procesos de acreditación que vaya consiguiendo cada organización.

PALABRAS CLAVE: Liderazgo. Autoevaluación institucional. Organizaciones educativas. Gestión participativa.

RESUMO: Este artigo descreve a influência positiva da liderança nos processos de autoavaliação institucional em uma organização que busca levantar um diagnóstico de gestão participativa, promovendo a colaboração de todos os agentes envolvidos, cujo objetivo é corrigir as fraquezas e necessidades detectadas e, em seguida, tomar decisões colegiadas e distribuidas. e acordado em beneficio das pessoas e, portanto, da organização. Para alcançar a gestão participativa, é necessário que a instituição possua uma metodologia de autoavaliação e, por meio de técnicas qualitativas e quantitativas, seja possível implementá-la periodicamente, de acordo com os processos de planejamento estratégico e credenciamento que cada organização está obtendo.

PALAVRAS-CHAVE: Liderança. Autoavaliação institucional. Organizações educacionais. Gestão participativa.

${ }^{1}$ Universidad Católica Silva Henríquez (UCSH), Santiago - Chile. Académico Licenciado en Educación. Editor de la Revista Foro Educacional. Profesional del Instituto Pastoral Juvenil de la Vicerrectoría de Identidad Estudiantil de la UCSH de Santiago de Chile. ORCID: https://orcid.org/0000-0003-0286-3515. E-mail: aberriosa@ucsh.cl

${ }^{2}$ Universidad Católica Silva Henríquez (UCSH), Santiago - Chile. Secretaria Académica Facultad de Educación. ORCID: http://orcid.org/0000-0003-4104-0116. E-mail: maravena@ucsh.cl

${ }^{3}$ Universidad de Granada (UGR), Melilla - España. Profesora Ayudante Doctora. Departamento de Didáctica y Organización Escolar. ORCID: https://orcid.org/0000-0002-5314-5639. E-mail: marinagc@ugr.es

${ }^{4}$ Universidad de Alcalá (UAH), Madrid - España. Profesor Titular de Universidad. Departamento de Ciencias de la Educación. ORCID: http://orcid.org/0000-0002-2775-0480. E-mail: mario.martin@uah.es 
ABSTRACT: This article describes the positive influence of leadership in the processes of institutional self-evaluation in an organization that seeks to raise a diagnosis of participatory management, promoting the collaboration of all agents involved, whose goal is to correct the weaknesses and needs detected and then make collegial and distributed decisions. and agreed on for the benefit of people and therefore the organization. To achieve participatory management, it is necessary that the institution has a self-assessment methodology and, through qualitative and quantitative techniques, it is possible to implement it periodically, according to the processes of strategic planning and accreditation that each organization is obtaining.

KEYWORDS: Leadership. Institutional self-evaluation. Educational organizations. Participative management.

\section{Introducción}

Los procesos de acreditación en la mayoría de las organizaciones suponen una excesiva carga de trabajo. Esto es debido a que se deben involucrar todas las áreas de la organización y conseguir que las personas, mientras desarrollan sus funciones diarias y habituales, entreguen informes, reportes, datos estadísticos, entre otros análisis, que demuestren lo que se ha realizado para lograr los objetivos estratégicos con el fin de generar una autoevaluación institucional.

El diagnóstico de la organización es determinar la radiografía actual de la organización y generalmente se desarrolla dependiendo de los años de acreditación que tiene la institución. La capacidad de autorregulación en este proceso es un buen hábito organizacional, el cual debe instalarse como un proceso continuo. Dicha autorregulación ayuda a estar en constante búsqueda de la calidad y de la mejora permanente, como también el tipo de liderazgo que ejercen las personas que están a cargo de llevar a cabo estos procesos complejos y de gran envergadura para la organización.

Lorenzo Delgado (2004) enfatiza el liderazgo con una visión compartida, dándole énfasis a la toma de decisiones colegiada y cooperativa y a que las responsabilidades residen en el grupo. Asimismo, Aravena y García-Carmona (2018) destacan las buenas relaciones humanas para generar cambios en el comportamiento de las personas y, por ende, lograr estos buenos hábitos es la gestión participativa que facilita la colaboración entre pares para tomar decisiones distribuidas de debilidades transversales que aquejan a la organización.

Por tanto, liderazgo, autoevaluación y gestión participativa, son los tres conceptos principales que se describen en este artículo, los cuales deben estar concatenados de manera sinérgica y, sobre todo, dirigirlos a la consecución de un diagnóstico realista y participativo, sin perder de vista que también hay otros elementos que se deben considerar cuando se trata de implementar una autoevaluación con fines de acreditación. 


\section{Liderazgo en organizaciones en proceso de autoevaluación}

Actualmente el ejercicio del liderazgo mantiene una estrecha relación con las organizaciones de éxito (GARCÍA-CARMONA, 2014). Es un tema que está de moda y se ha aplicado a muchas áreas de conocimiento, siendo la de las organizaciones educativas la que capte nuestra atención en este momento.

Debido a la amplitud conceptual del término liderazgo, surgen diversos tipos y campos de clasificación del mismo. En este sentido, Lorenzo Delgado (2004, p. 195-196) afirma que el líder no se entiende como una atribución individual o ambiental, siendo esto, más propio de un director o gerente, sino que, el liderazgo se conceptualiza como una "función, una cualidad y una propiedad que reside en el grupo y que dinamiza la organización [...] para generar su propio crecimiento en función de una misión o proyecto compartido". Con esta definición podemos observar cómo se llena de significado este término dotándolo de identidad propia. Teniendo en cuenta su significado, el autor añade como características que:

- $\quad$ Es una función: es una actividad llevada a cabo por el colectivo designado para ello. Por este motivo se utiliza más el término liderazgo que el término líder.

- $\quad$ Es estratégica para toda la organización: posee unas técnicas y conjunto de actividades para conseguir los objetivos de la organización y ayuda a elaborar una visión de la organización.

- $\quad$ Es compartida: no está representada por una única persona sobre la que recaen todas las decisiones, sino que se ejerce de manera colegiada y cooperativa.

- $\quad$ Se inserta en la cultura: no se trata de algo ajeno al contexto de la organización en el que se desarrolla, sino que forma parte de él.

- $\quad$ Reside en el grupo: en el ejercicio de la misma influirán tanto las características grupales como las personales de cada miembro.

El trabajo en equipo cobra aquí especial relevancia y es que se persiguen objetivos comunes por el grupo, se comparten visiones y experiencias vividas para lograr el "sueño compartido" mencionado por el autor. Asimismo, el contexto marcará significativamente el funcionamiento y la toma de decisiones de la organización, así como las particularidades de cada miembro dentro del grupo (GARCÍA-CARMONA, 2014).

De este modo los líderes deben tener la capacidad de transformar de manera positiva las actitudes y hábitos de comportamiento de las personas en función de los procesos de cambio, y también fomentar las buenas relaciones humanas; de lo contrario este proceso no tendrá sentido 
y carecerá de confiabilidad en el transcurso del tiempo (ARAVENA; GARCÍA-CARMONA, 2018). En este sentido, los procesos de autoevaluación cobran especial relevancia.

La autoevaluación llevada a cabo en una organización pretende reflexionar sobre las tareas y acciones realizadas durante un periodo de tiempo con el fin de mejorar el funcionamiento y los resultados de la misma. Este proceso, de vital importancia para el ejercicio de las organizaciones, ayuda a detectar fortalezas y debilidades que deben analizarse de manera reflexiva y colegiada para garantizar su adecuado desarrollo.

Para dicho proceso es importante contar con metodologías de autoevaluación y mecanismos de autorregulación efectivos, estableciéndose éstos como grandes desafíos de una entidad que aprende aunado capacidades, cualidades y actitudes que deben poseer las personas que lideran (GAIRÍN; MUÑOZ, 2008). De este modo un liderazgo basado en procesos de autoevaluación propia y de la organización contribuirá al desarrollo de la organización y a la consecución de sus objetivos.

\section{Autoevaluación institucional}

A lo largo de los años han sido varios autores quienes han definido la autoevaluación sometiéndose al devenir histórico. Por ende, el concepto ha ido evolucionando, ampliando y modificando las aportaciones al campo educativo. Es así como encontramos diversas definiciones según la perspectiva de cada autor y éstas se condicen con las estrategias de liderazgo que se implementan.

La primera lo hace centrada en una concepción como un proceso propio y comunitario. Figueroa y Muñoz (2014) conciben la autoevaluación como un proceso que implica el análisis y la crítica de manera colectiva. Esto se realizará por una organización con herramientas propias y regulado por sí misma. A raíz de lo anterior comprendemos que es un proceso interno de toda organización, cuya finalidad es analizarse a sí misma, de manera crítica de acuerdo a los objetivos propios de la organización.

Por su parte, Iranzo, Tierno y Barrios (2014) comprenden la autoevaluación institucional como "un proceso colegiado" (p. 251), reforzando la característica comunitaria de la autoevaluación. Es vista como una herramienta relevante para producir mejoras e innovaciones reales en la organización; además de definir el concepto de autoevaluación "como estrategia de diagnóstico para la toma de decisiones” (p. 237). Enfatizando la finalidad práctica y de proyección de la autoevaluación, nuevamente se realza el carisma comunitario de esta. 
La mirada de Landi y Palacios (2010) nos permite definir la autoevaluación como una herramienta que "trata de un recurso para objetivar los puntos reales de acuerdo y desencuentro, las fortalezas y las debilidades: construir un análisis de la situación inicial de cada [institución] frente a las nuevas necesidades y demandas sociales, políticas y educativas” (p. 165). La concepción de los autores es más bien una mirada interna que debe dialogar con su entorno, reflejando así a la autoevaluación como una objetivación de las necesidades del ambiente externo y cómo estas se subsanar o se atienden desde la cultura propia de la institución.

Silva Triviño (2002) nos presenta la autoevaluación como: "una forma de evaluación mediante la cual una unidad, programa, facultad o institución, reúne y analiza información sustantiva sobre la base de sus propósitos declarados y a la luz de un conjunto de estándares previamente definidos y aceptados" (p. 31). Genera una definición aún más relevante, en donde ahonda en la recopilación de datos, para ser analizados desde objetivos y estándares, lo cual lleva a evaluar la realidad desde la propia identidad, de lo que se declara ser. Desde esta mirada podemos comprender que la autoevaluación institucional requiere una clarificación previa y negociada sobre la finalidad, los requisitos y consecuencias de la actividad que se va a realizar en la institución.

Reforzando lo anterior, Iranzo, Tierno y Barrios (2014), llevan a la autoevaluación a la disciplina educativa, en donde demuestran que autoevaluación educativa es vista "como el proceso de recopilación sistemática y analítica, que implica el intercambio de datos relativos a los procesos educativos de individuos, grupos y organizaciones [...] con el fin de facilitar el aprendizaje entre todas las partes interesadas" (p. 182), quedando claro que, la autoevaluación educativa es un proceso de análisis de datos de la realidad que faciliten el aprendizaje de todos en la organización educativa.

Silva Triviño (2002) asume y propone que la autoevaluación en cuanto proceso, debe considerar lo siguiente: existir una fase o periodo de investigación, la cual ayude a comprender los objetivos e información de la institución. Otra fase es el análisis de la situación, procesos y resultados de una institución (unidad, programa o carrera). Se analizan documentos oficiales que declaren la misión y los objetivos de la institución (unidad, programa o carrera) y se agregan ciertos referentes o estándares de calidad, concibiendo que la autoevaluación tiene un "carácter estratégico, prospectivo y orientado al cambio" (p. 54). En consecuencia, el proceso de autoevaluación debe y se propone desde un plano que, de cuenta de una organización previa (objetivos trazados), y que la misma institución sea quien conduzca la autoevaluación; insistiendo y recalcando la participación de los actores pertinentes e involucrados. Admitiendo 
así la autoevaluación como una iniciativa propia y que busca evaluar sus aspiraciones y, por consiguiente, obtener una certificación externa.

En Chile la Ley, no 21.091, muestra a la autoevaluación institucional como un "proceso participativo mediante el cual la institución de educación superior realiza un examen crítico, analítico y sistemático del cumplimiento de los criterios y estándares definidos por dimensión, teniendo en consideración su misión y su proyecto de desarrollo institucional" (artículo, $\mathrm{n}^{\circ} 16$ ). La ley aglutina y consolida las concepciones presentadas anteriormente, en donde la autoevaluación se comprende como un proceso propio y comunitario, crítico y reflexivo que orienta el desarrollo de la institución (CHILE, 2018)

\section{La autoevaluación como un proceso articulado de gestión}

La autoevaluación se concibe como un proceso donde se deben identificar hitos que permitan comprender y planificar. El primero de ellos es la preparación y diseño del proceso. Esto demarca que debe ser gestado desde los participantes de la autoevaluación fijándose así fases y niveles de cumplimiento de un cronograma o programación a realizar. Otro hito significativo es la recopilación de la información, es decir, obtener datos que permiten comprender la consecución de los objetivos trazados con anterioridad, recogiendo evidencias que muestren el cumplimiento de la misión y plan de acción que se está ejecutando. Un tercer hito es la redacción del informe, el cual objetiva e identifica las debilidades y fortalezas de la institución. Por lo anterior se comprende que desde el informe nacen líneas de acción o consecuencias de acciones de gestión, las cuales se concatenan a un seguimiento, hasta que exista un nuevo proceso de autoevaluación (SILVA TRIVIÑO, 2002).

Por otro lado, se puede entender la autoevaluación desde una mirada cíclica, que permite identificar y comprender que la pertinencia de una autoevaluación es algo recurrente y sobre todo dinámico. Silva Triviño (2002) conceptualiza las fases de la autoevaluación dando a entender que ésta nunca se deja de lado o se encapsula a un momento en particular, se asemeja a una actividad constante de toda organización, más aún de una organización de educación superior, que debe responder a las necesidades de la cultura y la sociedad.

Landi y Palacios (2010) señalan "características puntuales de la autoevaluación” (p. 160), que se presentan a continuación:

No es algo externo, ya que los actores de la organización educacional son quienes conducen e implementan el proceso, además lo decreta la CNA (2006) “debe realizar un 
proceso de autoevaluación con participación de actores internos" (p. 17), la implicancia y participación es fundamental en el proceso.

Sí los actores tienen problemas de organización, ahí es posible el asesoramiento de personas externas, pero no se recomiendan.

El fin es la autorregulación de la institución, debido a que la finalidad es fortalecer la gestión institucional.

- Las dimensiones, aspectos y criterios utilizados para esta autoevaluación son previamente seleccionados por la propia institución, pueden ser plan de desarrollos institucionales u objetivos de corto, mediano y largo plazo.

- $\quad$ La finalidad de la autoevaluación es el informe de autoevaluación, el cual se concibe con acciones de mejora a ser implementadas para optimizar la calidad de la formación o de las nuevas funciones que se proponga la institución.

La CNA (2006) refuerza la idea de proceso ya que la engloba dentro de la gestión y conducción de una unidad organizativa que se autoimpone acciones de mejoramiento y seguimiento de su mejora constante. Lo establece de la siguiente manera:

La autoevaluación de una unidad forma parte de un proceso cíclico y permanente que proporciona información confiable para la toma de decisiones, permite realizar una planificación de procesos y acciones relevantes, organizarse para desarrollar dichas acciones y procesos y monitorear su evolución y cumplimiento (p. 10).

Al ser vista como proceso, la autoevaluación empodera a la institución o unidad para demostrar su capacidad de autorregulación. Particularmente, en el caso de las carreras de pregrado, al ejecutar el proceso de autoevaluación la CNA (2006) sentencia lo siguiente: la carrera reforzando la implicación fundamental de los actores internos; pero a su vez, "La carrera debe demostrar capacidad de realizar un análisis crítico de su realidad, en base a los criterios de evaluación establecidos" (p. 17). Este proceso crítico debe demostrar una presentación de desafíos claros a cumplir, siendo conscientes y muy lúcidos respecto a las herramientas, personal y presupuesto que se cuenta para cumplirlo. Más aún, debe estar en armonía con la gestión de la institución. El plan de acción "debe ser adecuado a la realidad de la unidad, demostrando que es consistente con sus propósitos y con el plan de desarrollo de la unidad y de la institución" (p. 23). Es por eso que se enmarca como un proceso y no como una actividad en particular.

Por esa razón Landi y Palacios (2010), invitan a comprender el proceso de autoevaluación "como la articulación de distintas fases complementarias de diseño, 
divulgación, ejecución, informe final y metaevaluación” (p. 176). Además, señalan que se requieren acciones de planificación recalcando la finalidad "como conclusión de los juicios de valor que provoca, se generen acciones de planeamiento institucional, a la vez que dinamiza la gestión institucional en un devenir dinámico que se retroalimenta constantemente” (p. 161162). Por tanto, es un proceso que se va retroalimentando y concibiendo como un sinfín, en otras palabras, es un trabajo constante.

Al comprender la autoevaluación como un proceso, al respeto Figueroa Céspedes y Muñoz Martínez (2014, p. 183) nos muestran tres grandes ventajas del proceso en sí mismo:

1) La autoevaluación "empodera" al colectivo, 2) Crea un sentido de pertenencia y compromiso y 3) Hace posible el diálogo entre todas las partes implicadas en la enseñanza y el aprendizaje, visibilizando procesos, de manera que permite una mayor coherencia entre lo que se necesita desde la perspectiva del alumnado y lo que se ofrece por parte del profesorado.

\section{Metodología de autoevaluación}

Las instituciones que se someten a procesos de acreditación requieren contar con metodologías participativas para autoevaluarse tanto institucionalmente como en la autoevaluación de carreras.

De acuerdo con los resultados de Fernández (2013) en una investigación sobre gestión participativa, menos del 50\% de los docentes perciben que el colegio les da la oportunidad de opinar e influir en el ámbito laboral. Asimismo, los mismos docentes piensan que la gestión participativa es una variable positiva que influye en el campo laboral, porque los hace más eficientes, aumenta el grado de compromiso y los hace más productivos. Es decir, perciben que la participación sí puede hacer un cambio en la mejora organizacional porque tienen la oportunidad de contribuir en la toma de decisiones.

La gestión participativa es una dimensión clave en estos procesos, dado que los diversos agentes deben involucrarse y colaborar en la autoevaluación (RODRÍGUEZ, 2018). Desde el interior de la organización son los primeros que también deben adaptarse, para que las mejoras sean efectivas y logren implementarse. Por otro lado, la participación por medio de aplicación de técnicas cualitativas y cuantitativas facilita la recolección de información, datos estadísticos, registros, etc., que luego se traducen en fortalezas y debilidades que surgen desde la mirada de diferentes actores. Es por ello que mientras más personas participen de forma planificada, más enriquecedor y real será el diagnóstico levantado. Por tanto, la radiografía de la institución es 
esencial para poder elaborar un plan de mejora, documento final que plasma todas las acciones, proyectos y/o actividades que se desarrollarán tanto en el corto, mediano o largo plazo.

A continuación, se presenta un cuadro comparativo que describe las diferencias y semejanzas entre ambas técnicas, con el fin de establecer sus bondades y criterios para determinar cuál de ellas aplicar dentro de un proceso de autoevaluación participativo.

Tabla 1 - Diferencias y semejanzas entre técnicas cualitativas y cuantitativas

\begin{tabular}{|c|c|c|}
\hline Técnicas & Diferencias & Semejanzas \\
\hline $\begin{array}{l}\text { Técnicas } \\
\text { Cualitativas }\end{array}$ & $\begin{array}{l}\text {-Focalizar las fortalezas y debilidades con un } \\
\text { pequeño grupo de personas. } \\
\text {-Levantar problemáticas específicas por área de } \\
\text { desempeño o departamento. } \\
\text {-Aplicación de categorías y subcategorías para } \\
\text { analizar las textualidades. }\end{array}$ & $\begin{array}{l}\text {-Ambas técnicas permiten } \\
\text { recolectar información para } \\
\text { el proceso. } \\
\text {-Ambas técnicas favorecen } \\
\text { la gestión participativa. }\end{array}$ \\
\hline $\begin{array}{l}\text { Técnicas } \\
\text { Cuantitativas }\end{array}$ & $\begin{array}{l}\text {-Levantar fortalezas y debilidades con grandes } \\
\text { muestras (alumnado, docentes, egresados, } \\
\text { empleadores) } \\
\text {-Aplicación de la estadística. Entrega datos } \\
\text { numéricos, frecuencias y porcentajes los que } \\
\text { son fácil de proyectar para analizar la } \\
\text { información. }\end{array}$ & \\
\hline
\end{tabular}

Fuente: Elaboración propia

\section{Reflexiones finales}

Las demandas de la sociedad actual requieren una nueva perspectiva de la evaluación de las organizaciones. El liderazgo ejercido en dichas organizaciones debe estar fundamentado en procesos deliberativos basados en una reflexión crítica y un análisis de la realidad para evaluar ideas y problemas. Habrá también de preocuparse por el bienestar de los otros y de lo que es bueno para todo el grupo, tomando conciencia de la dignidad y derechos de los demás (GARCÍA-CARMONA, 2015). Asimismo, el liderazgo institucional debe generar y propiciar espacios de gestión participativa, con el fin de que la toma de decisiones sea lo más acertada, compartida y colegiada, según las necesidades y requerimientos de la organización.

De este modo los procesos de autoevaluación cobran especial relevancia al incorporar una metodología para reflexionar sobre las acciones realizadas y permitir así una mejora en el desarrollo organizacional. Mediante estos procesos de análisis en profundidad del funcionamiento de las organizaciones, los líderes favorecen su mejora y promueven un clima positivo. 
Por lo tanto, autoevaluación y gestión participativa, son dos aspectos que deben ir aunados cuando se requiere levantar un diagnóstico, porque según las evidencias de algunos estudios, la participación mejora la productividad, como también el clima organizacional. La institución debe planificar estrategias que generen espacios para que las personas sientan que aportan desde su experiencia y conocimiento desde la práctica.

Por último, los responsables de acreditación deben tener presente estos conceptos y ser apoyados por las autoridades para poder implementarlos. De lo contrario es compleja su aplicación. La participación colaborará de este modo en una toma de decisiones distribuida (FERNÁNDEZ, 2013).

\section{REFERENCIAS}

ARAVENA, M.; GARCÍA-CARMONA, M. Liderazgo ético y transformacional en organizaciones educativas en proceso de cambio (CIOIE). 2018.

BELTRÁN, M.; FERNÁNDEZ, A. Gestión del conflicto ¿Qué hacemos ahora? Santiago de Chile: Ediciones UCSH, 2018.

CHILE. Ministerio de Educación. Ley 21.091, 29 de mayo de 2018. Sobre educación superior. Disponible en: https://www.leychile.cl/Navegar?idNorma=1118991. Acceso en: 10 sep. 2020 .

CHILE. Ministerio de Educación. Ley n. 20.129, 17 de septiembre de 2006. Establece un sistema nacional de aseguramiento de la calidad de la educación superior. Disponible en: https://www.cnachile.cl/Documentos\%20de\%20Paginas/Ley_Aseg_Calidad\%2020.129.pdf. Acceso en: 10 sep. 2020.

CNA. Comisión Nacional de Acreditación. Guía para la Autoevaluación Interna acreditación institucional Universidades. Santiago, 2006. Disponible en: https://www.cnachile.cl/SiteAssets/Lists/Acreditacion\%20Institucional/AllItems/Gui\% $\%$ CC\%8 1a\%20para\%201a\%20autoevaluacio $\%$ CC $\% 81$ n\%20interna $\% 20$ Universidades.pdf. Acceso en: 10 sep. 2020.

FERNÁNDEZ, A. Redefinición de procesos y estructura organizacional a través de la gestión del cambio en una institución educativa. 2013. Tesis (Doctoral) - Universidad de Sevilla, 2013.

FIGUEROA CÉSPEDES, I.; MUÑOZ MARTÍNEZ, Y. La guía para la inclusión educativa como herramienta de autoevaluación Institucional: reporte de una experiencia. Revista Latinoamericana de Educación Inclusiva, v. 8 n. 2, p. 179-198, 2014.

GAIRÍN, J. El aprendizaje en las organizaciones. In: GAIRÍN, J. (Coord.). Aprendizaje situado y aprendizaje conectado. Implicaciones para el trabajo. Madrid: Wolters Kluwer, 2016. capítulo I. 
GAIRÍN, J.; MUÑOZ, J. Agente de cambio en el desarrollo de las organizaciones.

Enseñanza, v. 26, p. 187-206, 2008.

GARCÍA-CARMONA, M. Análisis de las percepciones sobre liderazgo y participación de las familias en asociaciones de madres y padres en contextos multiculturales. Un estudio comparativo entre Nueva York y Granada. Tesis (Doctoral) - Universidad de Granada, España, 2014.

GARCÍA-CARMONA, M. La educación actual: retos para el profesorado. Revista IberoAmericana de Estudos em Educação, v. 10, n. 4, p. 1199-1211, out./dez. 2015. DOI: https://doi.org/10.21723/riaee.v10i4.8262

IRANZO, P.; TIERNO, J. M.; BARRIOS, R. Autoevaluación institucional y dirección de centros inclusivos. Teoría de la Educación. Revista Interuniversitaria, v. 26, n. 2, p. 229$257,2014$.

LANDI, N.; PALACIOS, M. E. La autoevaluación institucional y la cultura de la participación. Revista Iberoamericana de Educación, v. 53, p. 155-181, 2010. DOI: https://doi.org/10.35362/rie530562

LORENZO DELGADO, M. La función de liderazgo de la dirección escolar: una competencia transversal. Enseñanza, v. 22, p. 193-211, 2004.

RODRÍGUEZ, D. Gestión organizacional. Santiago de Chile: Ediciones UC, 2018.

SILVA TRIVIÑO, M. Hacia la calidad de la educación universitaria: principios y mecanismos. Contextos: Estudios De Humanidades Y Ciencias Sociales, v. 9, p. 23-35, 2002.

\section{Cómo referenciar este artículo}

BERRIOS, A.; ARAVENA, M.; GARCÍA-CARMONA, M.; MARTÍN BRIS, M. Liderazgo y autoevaluación institucional. Revista Ibero-Americana de Estudos em Educação, Araraquara, v. 15, n. esp. 4, p. 2600-2610, dez., 2020. e-ISSN: 1982-5587. DOI: https://doi.org/10.21723/riaee.v15iesp4.14508

Remitido el: 10/09/2019

Revisiones requeridas el: 10/01/2020

Aprobado el: $30 / 04 / 2020$

Publicado el: 01/12/2020 